\title{
Zum Beispiel Nyegezi: Möglichkeiten und Probleme einer Journalistenschule in Afrika
}

\author{
von Piet A. Winnubst
}

Die erste Ausbildungsmöglichkeit für Journalisten in Afrika wurde 1937 in Agypten an der American University in Kairo geschaffen. Die erste Journalistenschule in BantuAfrika, südlich der Sahara und nördlich der Südafrikanischen Union, entstand 1963 in Mwanza-Nyegezi am Südufer des Viktoria-Sees in Tanzania. Obwohl die katholischen Initiatoren mit Recht stolz sein können auf diese Gründung und auf den damit geleisteten Beitrag zur Entwicklung der Publizistik in Tanzania, Uganda, Kenia, Malawi und Zambia, bleibt der späte Zeitpunkt ebenso bedauerlich wie symptomatisch. Seit dieser Gründung ist kein Jahr vergangen, in dem nicht über die Planung oder die Eröffnung weiterer Kurse, Schulen oder Institutionen zur Ausbildung von Journalisten in Afrika berichtet wurde. ${ }^{1}$

Uber die augenblickliche Situation, über die Anzahl und das Leistungsniveau der verschiedenen Ausbildungsmöglichkeiten liegen nur unzureichende Daten vor. ${ }^{2}$ Außerdem fehlen Angaben über den vorhandenen oder zu erwartenden Bedarf an qualifiziertem Personal in den einzelnen Medien wie Presse, Hörfunk und Fernsehen. Allenfalls ist in den beiden führenden Presseländern Schwarzafrikas, in Kenia und Nigeria, ein verläßlicher Uberblick zu gewinnen. ${ }^{3}$ Der ansonsten negative Befund macht detaillierte Angaben über die Notwendigkeit weiterer Ausbildungsmöglichkeiten sowie über den Umfang solcher Institutionen oder die Art des regional erforderlichen Lehrprogramms unmöglich. Daß zur Zeit in Afrika ein großer Bedarf an qualifizierten Journalisten besteht, wird jedem ersichtlich, der die Entwicklung in diesem Kontinent mit Interesse verfolgt.

\section{Kommunikation als Katalysator der Entwicklung}

Das Problem der Ausbildung von Afrikanern in der Handhabung moderner Massenmedien hat Dimensionen, die über den Rahmen spezialisierter Kommunikationsarbeit hinausreichen. Nicht nur die großen Kommunikationsmedien werden in ihrer afrikanischen Ausprägung beeinflußt von den Nachwirkungen einer mehr intuitiven Rede- und „Palaver"-Kultur. Mehr noch leidet die gesamte sozio-ökonomische Entwicklung unter diesem Erbe, dessen kultureller Eigenwert unbestritten ist. Paradoxerweise erschwert

Piet A. Winnubst, Diplom-Journalist und Kunsthistoriker (Niederlande), arbeitete von 1961 bis 1966 für die Entwicklung der Presse in Afrika. Er gründete und leitete als Direktor die erste Journalistenschule in Bantu-Afrika, die 1963 in Mwanza-Nyegezi in Tanzania errichtet wurde. Seit 1966 ist der Verfasser unseres Beitrags beim Bischöflichen Hilfswerk "Misereor" als Referent für mehrere afrikanische Länder tätig. 
dieses Erbe jedoch die Ausbildung von ausreichendem Personal mit der jeder Intuition zuwiderlaufenden Eignung zur schriftlichen Fixierung und Mitteilung von Sachverhalten.

Das Entwicklungsproblem der Medienpublizistik wie des sozial-wirtschaftlichen Fortschritts ist gleichermaßen ein Entwicklungsproblem der menschlichen Fähigkeit, Probleme zu analysieren, Ideen logisch zu verarbeiten, in Aussagen zu kleiden und anderen mitzuteilen. Solche Fähigkeiten werden nicht nur von Journalisten verlangt. Sie sind Voraussetzung für jeden Berufsstand, der an der sozialen Integration seiner Gesellschaft durch Anregung zu aktiver Kommunikation beitragen will.4 In der Journalistenschule werden solche Fähigkeiten erlernt. Gerade in den Entwicklungsländern, wo man intensiv nach neuen, den ethnischen Voraussetzungen angepaßten Strukturen sucht, und wo der Bedarf an qualifizierten Kräften in fast allen für den sozialen, wirtschaftlichen und kulturellen Fortschritt wichtigen Bereichen noch lange Zeit sehr groß sein wird, erscheint es notwendig, jede Fachkraft mit den Anfangsgründen des journalistischen Handwerks: dem Beobachten, Analysieren, Sich-mitteilen-Können, vertraut zu machen. Für die Zukunftschancen afrikanischer Publizistik, die aus rein wirtschaftlichen Gründen auf Jahre hinaus keinen durchgegliederten journalistischen Berufsstand unterhalten kann, ist es unerläßlich, das Potential der im sozialen Bereich tätigen Kräfte für freie Mitarbeit zu nutzen. Insbesondere die wenigen afrikanischen Akademiker und ausgebildeten Fachleute in den verschiedenen entwicklungswichtigen Bereichen sollten angehalten werden, Entwicklungsförderung durch Unterstützung eines besseren und freieren Kommunikationsflusses in ihrer Gesellschaft zu betreiben.

Obwohl man heute den Entwicklungsstand einer Gesellschaft an der Funktionsfähigkeit ihrer intermedialen Kanäle messen kann, gilt es doch ebenfalls als gesichert, daß selbständiges Rezipieren von Massenkommunikation auf dem Wege zwei- und mehrstufigen Meinungs- und Erfahrungsaustausches vorbereitet werden muß. In der interpersonalen Begegnung liegt auch die größere Möglichkeit zum Abbau sozialer WertHierarchien, der u. a. erst eine intensivere Wirkung der Massenmedien bedingt. ${ }^{5}$

Aus diesen Gründen übersteigt die Bedeutung journalistischer Ausbildung in einem Entwicklungsland den relativ eng gefaßten Rahmen eines Beitrags zur Entwicklung der Publizistik. Die hier geleistete Arbeit wird zu einem der wesentlichen Instrumente moderner Entwicklungsförderung überhaupt.

\section{Africans should write more}

Es ist eine bedauerliche Tatsache, daß sich nur wenige Afrikaner nach Beendigung ihrer Studienzeit noch intensiv intellektuell weiterbilden. Die beste Methode dazu, die der Verfasser den Absolventen der von ihm geleiteten Journalistenschule empfahl, war: to write more. Das Schreiben entwickelt kreatives Denken, es zwingt zu Definitionen, wo das Gefühl Umschreibungen sucht, es führt zur Analyse von Ideen und Erfahrungen, zur bewußten Festlegung von Zielen und Methoden. Der gebildete Afrikaner ist „Kind zweier Welten. Sein kultureller Hintergrund steht in ständigem Widerspruch zum kulturellen Hintergrund seines Tätigkeitsgebietes, ja, seines eigenen Tuns. Er findet sich täglich und stündlich in einer Doppelrolle. "

Jeder afrikanische Student sollte lernen, mit der Feder in seiner Hand zu denken. Der spätere intellektuelle Abfall der Akademiker ist alarmierend. Nur wenige bemühen sich, Erfahrenes und Erlerntes schriftlich mitzuteilen, und viele lesen kaum noch etwas. 
Die Ursachen dazu liegen im psychologischen Klima der Entwicklungsländer und in dessen Einfluß auf das Erziehungssystem. Die so sehr angestrebte sozio-kulturelle Entwicklung ist abhängig von wirtschaftlichen Sachzwängen; „Entwicklung“ wird in Prozentzahlen aufgerechnet und Lebensfreude mit ökonomischen Kategorien definiert. $\mathrm{Da}$ Sozialversicherungen und Altersversorgungen fehlen, hat der Student davon auszugehen, daß er persönlich und sein angestrebtes finanzielles Einkommen die Rentenbasis für Eltern und Verwandte darstellen. Akademische Grade gelten vor diesem Hintergrund „als Freipaß zum hohen Gehalt, zum ,executive salary'. Das ist begreiflich, fördert aber die Tendenz zum Pauken, Bluffen und Vergessen" 7 . Hinzu kommt, daß der afrikanische Akademiker in seinem Beruf meist eine Einsamkeit erfährt, die der gewohnten Gruppenbindung widerspricht. Die tägliche Arbeit erfordert seine ganzen Kräfte. Eine intensive Kommunikation mit geistig interessierten oder auf gleichem Niveau stehenden Freunden ist selten möglich.

Es wäre ein Mißverständnis, aus der hier gegebenen Darstellung der Situation die Behauptung ableiten zu wollen, daß nur durch eine intensive Vermittlung journalistischer Fähigkeiten das Problem der Entwicklung in Afrika gelöst werden könne. Solche Argumente für die Notwendigkeit der Gründung von journalistischen Ausbildungsstätten legen nur zusätzlich dar, daß es nicht nur sinnvoll wäre, die Errichtung von berufsbildenden Journalistenschulen, sondern darüber hinaus alle Ausbildungsprogramme in entwicklungswichtigen Bereichen zu fördern, die eine journalistische Schulung ergänzen.

\section{Nyegezi: Gründung einer Journalistenschule in Tanzania}

Schon 1956 hatten sich die tanzanischen Bischöfe entschlossen, die Ausbildung von einheimischen Journalisten zu fördern; aber vorerst geschah nichts. Auf der Suche nach Möglichkeiten in Dar-es-Salaam, Nairobi, Tabora und Entebbe nahm man Kontakt auf mit P. John Lavoie, dem Leiter des gerade zu dieser Zeit von den Weißen Vätern in Nyegezi bei Mwanza, am Südufer des Viktoria-Sees, gegründeten „Social Training Centre" zur Ausbildung von afrikanischen Führungskräften. ${ }^{\circ}$ Dieses bereits nach kurzer Tätigkeit zu Ansehen gelangte Zentrum bot sich an, eine Journalistenschule als spezielle Sektion zu integrieren. Die von dem niederländischen Verleger Dr. C. Verhaak zur Förderung afrikanischer Kommunikationsmedien geschaffene Institution „African Publicity " half mit, die personellen Voraussetzungen zu schaffen. Dazu zählte auch die Verpflichtung des Verfassers als ersten Direktors des „Publicity Media Institute“.

Trotz der bischöflichen Approbation fehlte es anfangs nicht an der Opposition kirchlicher Stellen gegen das Vorhaben, die sowohl diözesan-politisch wie ideologisch begründet wurde. Diözesan-politisch: Manche Bistumsverwaltung wünschte sich die Schule in ihrem Einflußbereich; ideologisch: die weniger der traditionellen Pastoral als vielmehr einer für vorkonziliare Zeiten recht weltoffenen Seelsorge verschriebene Lehrtätigkeit

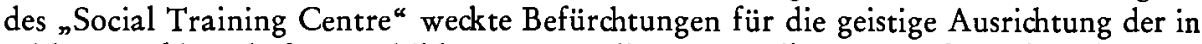
solcher Nachbarschaft ausgebildeten Journalisten. Im allgemeinen fand der Plan eine günstige Resonanz. Für den Erfolg der Schule war es jedoch entscheidend, die Mitarbeit nichtkatholischer Gruppen in der Gesellschaft sicherzustellen: der Regierung, der Presse und anderer christlicher Kirchen.

Die Regierung förderte das Engagement. In den letzten Monaten vor der Unabhängigkeit, die in Tanzania im Dezember 1961 verkündet wurde, fürchteten viele der im 
Lande lebenden Europäer um ihre persönliche Sicherheit. Hinzu kam die Besorgnis über eine mögliche Verschlechterung der wirtschaftlichen Situation. Zahlreiche Ausländer verließen die ost- und zentralafrikanischen Länder. Mit Erfolg warben die jungen afrikanischen Politiker in der ersten Zeit nach der Unabhängigkeit um das Vertrauen der ehemaligen Kolonisten. So wurde verständlicherweise die von einem Europäer vorgetragene Initiative zur Gründung einer Journalistenschule offiziell mit größtem Interesse registriert. Am 23. Januar 1963 traf eine Regierungsdelegation mit einer Sondermaschine aus Dar-es-Salaam in Mwanza/Nyegezi ein, um die Schule feierlich zu eröffnen.

Die Haltung der nationalen Presse, die fast ausschließlich mit britischen Journalisten arbeitete, war neutral bis reserviert. In einzelnen Fällen trat ein anti-katholisches Ressentiment zutage, und im allgemeinen war man von der $Z_{\text {weckmäßigkeit einer }}$ solchen Nachwuchsförderung nicht überzeugt: "Journalists are trained on the job!" In Tanzania gab es am Tag der Unabhängigkeit nur drei ausgebildete einheimische Journalisten, von denen zwei gleich Regierungsämter übernahmen. In den übrigen Ländern aus dem Einflußbereich des „Publicity Media Institute“, in Uganda, Malawi und Zambia, war es nicht wesentlich besser, wobei man Kenia einen Sonderstatus zubilligen müßte.

Die Arbeit der anderen christlichen Kirchen war auf dem Gebiet der Pressepublizistik in diesen Ländern relativ gering. Untereinander rivalisierten die einzelnen Denominationen zu sehr, als daß sie für die Trägerschaft der Schule in Betracht gezogen werden konnten.

\section{Die kommunikationspolitische Situation}

Noch zu Beginn der sechziger Jahre hatte die Kirche überwiegend ein pastoral-missionarisches Interesse an der Entwicklung der Kommunikationsmittel in Afrika. Das Verständnis für publizistisches Handeln und publizistisches Funktionieren spiegelte weithin den Wissens- und Uberzeugungsstand europäischer Missionsseminare, der zumeist nocb im 19. Jahrhundert wurzelte. Die Intention publizistischer Aussage hatte kämpferisch zu sein, die Wirkung sich im Wandel der Meinungen, wenn nicht gar im Taufregister auszudrücken. Dem Verfasser wurde seine Aufgabenstellung in Afrika zu Beginn seiner Tätigkeit umschrieben als "to investigate possibilities of helping the development of the Catholic press in East and Central Africa - to stimulate all those activities which directly or indirectly help the spreading of the Christian message through the printed word“. Die afrikanische Reaktion auf solche Einstellung wurde am deutlichsten vom damaligen Informationsminister von Malawi während eines Gesprächs formuliert, in dem ihm der Verfasser anbot, Journalisten für sein Ministerium auszubilden: „I smell Vatican imperialism in your enterprise."

Niedriges Alphabetisierungsniveau, geringe Kaufkraft der Bevölkerung und eine schlecht entwickelte Infrastruktur führten damals in den genannten Ländern schon bald dazu, daß der Zeitungsmarkt ausgelastet war. Am Totalangebot waren die katholischen Zeitungen und Zeitschriften nur mit 6 v. H. beteiligt. Ein Wachstum des Marktanteils mußte aus verschiedenen Gründen als unmöglich angesehen werden:

1. Es widersprach der Mentalität wie der Vorbildung der zuständigen kirchlichen Stellen, Kommunikationsmedien als Mittel zur sozio-ökonomischen und sozio-kulturellen Integration der Gesellschaft zu sehen, für die sie sich moralisch verantwortlich glaubten. Sie schätzten vor allem die Presse als zusätzliches Instrument der Indoktrinierung und 
Missionierung, wobei die einzelnen Missionare noch ihre individuellen, von den Heimaterfahrungen geprägten deutschen, englischen, italienischen, spanischen oder niederländischen Vorstellungen mitbrachten.

2. Ein praktisches Problem erwies sich als nicht weniger gravierend: Obwohl eine ausreichende Anzahl von Druckereien und Druckern, vor allem für religiöse Broschüren und Schulbücher, zur Verfügung stand, fehlte es an qualifizierten Kommunikatoren. Mehr noch machte sich der Mangel an Managern und Wirtschaftsfachleuten bemerkbar, mit ausreichenden organisatorischen und administrativen Kenntnissen, um z. B. das Vertriebsnetz einer Zeitung aufbauen zu können.

Das Mentalitätsproblem ist im Grundsatz bis heute nicht behoben. Hier gilt es abzuwarten, bis der Afrikanisierungsprozeß der Kirche weiter fortgeschritten ist und sie innerhalb der sich immer noch ständig ändernden Strukturen in den einzelnen Ländern des Kontinents jenen Platz gefunden hat, der es ihr gestattet, eine auf den notwendigen Dialog mit der Gesellschaft eingestellte Publizistik zu entwickeln.

Aus diesen Gründen zog sich der Verfasser bald von einer an und mit afrikanischen katholischen Publikationen geleisteten Arbeit zurück und konzentrierte sich ganz auf die Ausbildungstätigkeit. Hier lagen die größeren Chancen für die Zukunft. In einem Brief an den Auftraggeber vom November 1961 begründete er diese Konzentration auf das Personalproblem: "Whatever the future structure of the (catholic) press will be, they will need trained personnel."

\section{Das Ausbildungsprogramm}

Gleich zu Beginn der Lehrtätigkeit im „Publicity Media Institute“ stellte sich das Problem einer sinnvollen Auswahl der an der Kursteilnahme interessierten Kandidaten. Man kam überein, daß Studenten mit einem Stipendium der Regierungen der beteiligten fünf Staaten (zusätzlich zeigte sich auch Nordrhodesien interessiert) oder Schüler, die von privaten Organisationen empfohlen wurden, von diesen Trägergruppen auch auf ihre Eignung geprüft wurden. Interessenten, die sich privat bewarben, mußten vor dem Lehrstab ihre Befähigung nachweisen.

Verlangt wurde ein reiferes Alter, vorausgesetzt eine zwölfjährige Schulbildung und - wenn eben möglich - einige Schreiberfahrung sowie gute Englischkenntnisse. Verheiratete Studenten konnten ihre Familien mitbringen und erhielten eine kleinere Wohnung zugewiesen. Pro Kurs sollten höchstens 15 Kandidaten aufgenommen werden, und zwar nach Möglichkeit drei aus jedem der beteiligten Länder: Kenia, Tanzania, Uganda, Malawi und Zambia.

Die Dauer der Ausbildung betrug 18 Monate, unterteilt in fünf verschiedene Zeitabschnitte mit etwa 1600 Ausbildungsstunden. Nach dem dritten "Term" entschied eine Zwischenprüfung über den weiteren Verbleib des Studenten am Institut. Waren die Leistungen eines Teilnehmers ungenügend, hatte er den Kurs zu verlassen. Seiner „sponsoring organisation" wurde ein detaillierter Bericht zugestellt. Im eigentlichen Lehrprogramm wurde Wert darauf gelegt, daß der Kandidat „needs some academical background". Man legte daher ein wohl abgewogenes Verhältnis zwischen „Professional training" und "General education" fest: im ersten Jahr 47 v. H. zu 53 v. H. und im zweiten Jahr 56 v. H. zu 44 v. H. Zur Ausbildung der künftigen Journalisten gehörte die Fertigung einer Wochenzeitung in eigener Regie, der "Nyegezi Weekly News“.

Der Lehrplan sah für die eigentliche Berufsausbildung (Professional training) folgende Fächer vor: Newes reporting: Theorie und Praxis der aktuellen Berichterstattung (Inter- 
views, Recherchieren, Nachrichten verfassen); Editing: Redigieren, Auszeichnen, Layout; Article writing: Schreiben von Leitartikeln, Features, Suche nach Affinität zum Publikum; History of the press: Entwicklung der Drucktechnik, Entwicklung der Zeitung, Geschichte der Pressefreiheit; Science of communications: Einführung in die Kommunikationswissenschaft, Propagandatechniken, Gibt es eine „öffentliche Meinung"?, Einfluß der Anzeigen auf die Publizität, Public Relations; Typewriting: tägliche Ubung; Photography: Theoretische Kenntnisse obligatorisch, praktische Ubungen für Studenten mit Kamera, Dunkelkammer vorhanden. ${ }^{10}$

Die bei den geringen Vorkenntnissen afrikanischer Journalisten als unerläßlich angesehene Vermittlung von Allgemeinbildung (General education) gliederte sich in folgende Fächer: Social etbics: Rechte und Pflichten des Menschen, der Mensch als Sozialwesen, als Individuum, eingegliedert in Familie und Gesellschaft, das Naturgesetz; Sociology: Die sozialen Beziehungen in Gesellschaft, Familie und Gemeinschaft; Economics: Einführung in die Gesetze der Güterproduktion und der Güterverteilung; Community development: Theorie des gesellschaftlichen Wandels in Entwicklungsländern; Co-operative movement: Geschichte und Prinzipien; Labour movement: Geschichte, Begründung, Ziele und Methoden der Arbeiterbewegung, Gewerkschaften; Political science: Regierung, Politik und der Staat, verschiedene Formen der Demokratia, die politische Partei, allgemeine Wahlen, Verfassung, immer im Blick auf afrikanische Verhältnisse; Community service: körperliche Arbeit im Institutsgelände. ${ }^{11}$

Je nach Leistungsstand erhielten die Teilnehmer nach Abschluß der Studien entweder ein Abschlußzeugnis, ein Diplom oder ein Diplom mit dem Vermerk besonderer Auszeichnung. Diese Papiere stellten eine Art Versicherungspolice dar. Sie waren auf dem Kommunikationsmarkt gefragt und öffneten überdies die Türen zu Regierungsämtern. Heute sitzen Absolventen des "Publicity Media Institute“ an verantwortlicher Stelle in vielen Redaktionen und Funkhäusern des ost- und zentralafrikanischen Raumes. Private Initiative hat hier einen Personalnotstand beseitigt, der sich für die Entwicklung der betroffenen Länder hätte katastrophal auswirken können. Das innerafrikanische Kommunikationsgefälle war ohnehin alarmierend. 1959 wurde in Malawi die erste Zeitung des Landes vom Führer der Malawi Congress Party, Aleke Banda, gegründet und auf einer Vervielfältigungsmaschine hergestellt. Zur gleichen Zeit übernahm in Nigeria bereits ein 27 jähriger Afrikaner die Chefredaktion einer großen, auf modernsten Rotationsmaschinen gedruckten Massenzeitung. ${ }^{12}$

\section{Nyegezi zwischen 1963 und 1970}

Inzwischen hat sich auch im "Publicity Media Institute“ vieles geändert. Das afrikanische Selbstbewußtsein - zu dessen Hebung die Schule beigetragen hatte - ist gewachsen. Hilfe von außen wird nicht mehr uneingeschränkt begrüßt. Kürzlich wurde dem Verfasser die jüngste Nummer der oben erwähnten, von den Studenten des Trainingszentrums redigierten und herausgegebenen „NSTC-News" zugestellt. Das Blatt erschöpft sich längst nicht mehr in mehr oder weniger gelungenen Schreibübungen. Seine Aussage wie sein Stil sind politischer, aggressiver geworden.

Der Leitartikel berichtet über den neuen Vorstand des Nyegezi Social Training Centre, in den neben vier Vertretern des Lehrpersonals (insgesamt zwei Geistliche und zwölf Laien, vier davon Afrikaner) drei Repräsentanten des tanzanischen Erziehungsministeriums und drei Personen „from the general public“ gewählt werden sollten. Damit wurde auch die Journalistenschule, wie seit Anfang 1970 alle Privatschulen in Tan- 
zania, unter den direkten Einfluß der Regierung gestellt. Geleitet wird das Zentrum allerdings weiter vom tanzanischen Geistlichen P. Mtabi.

Es ist zur Zeit noch nicht abzusehen, inwieweit die geschilderte Entwicklung Einfluß auf den internationalen und den christlichen Charakter der Schule nehmen wird.

Der Verfasser hat das Ausbildungszentrum vor vier Jahren verlassen, die Verbindung aber aufrechterhalten. So ist er über die steten Strukturänderungen informiert. Sie kommen für ihn nicht unerwartet. Die Integration der als westlicher Entwicklungsbeitrag gedachten Schule in die sich rasch entwickelnde Struktur des Landes sowie die technischen Verbesserungen des Lehrprogramms in den letzten vier Jahren sind in jedem Fall positiv zu beurteilen. Es ist nicht zulässig, diesem Wandel westliche Begriffsmuster wie Nationalisierung, Gleichschaltung, Vernichtung des Privateigentums etc. als Hilfen der Motivanalyse zu unterlegen. Die afrikanische Wirklichkeit entwickelt sich nach anderen Kategorien.

\section{Zusammenfassung und Ausblick}

Die erste Dekade nach dem zweiten Weltkrieg (evtl. noch bis 1960) erwies sich als ideal für die Gründung neuer Zeitungen und Zeitschriften in Afrika. Demgegenüber boten die ersten Jahre nach den Unabhängigkeitserklärungen die besten Chancen zur Errichtung von Institutionen aller Art, die dem Mangel an Personal, vor allem an qualifizierten Journalisten, abzuhelfen suchten.

In den letzten Jahren haben die verschiedenen Regierungen zu ihrem eigenen Stil gefunden. Das wesentliche Streben zielte darauf, eine vom Kolonialerbe abgelöste ideologische Basis zu finden, in der sich der afrikanische Mensch nach landeseigenen Bedürfnissen sozio-ökonomisch und kulturell entfalten konnte. Diese Entwicklung mußte $z$ wangsläufig zu engeren Beziehungen der afrikanischen Länder untereinander führen. Mit solchem Selbstbewußtsein wächst die Schwierigkeit, von außen mit der Gründung von Institutionen wie Journalistenschulen Einfluß auf die Ausbildung des Nachwuchses zu nehmen. War früher hauptsächlich auf die Differenzen zwischen englischer und französischer Mentalität bei solchem Vorhaben Rücksicht zu nehmen (englisch: journalists are trained on the job; französisch: Ausbildung im Kolonial-Mutterland), so hat man sich heute mit neuen, afrikanischen Ideologien auseinanderzusetzen. Jedes externideologische, z. B. europäisch-katholische Engagement wird auf große Widerstände stoßen.

Außerdem erscheinen prinzipiell Versuche zur Gründung von journalistischen Ausbildungsstätten in Afrika nur dann noch sinnvoll, wenn folgende Voraussetzungen erfüllt werden:

Das Institut soll, soweit wie möglich, nur fachtechnische Kenntnisse vermitteln und als Berufsschule arbeiten.

Das Institut soll unter der Leitung und unter der Verantwortung von einheimischen Kräften stehen.

Das Institut soll im Rahmen einer schon bestehenden, nationalen Ideologien und Entwicklungsvarianten zugewandten Institution aufgebaut werden. Kriterien allgemeiner Art wie die Berücksichtigung der nationalen Kommunikationssituation und ihrer personellen Bedarfslage werden als selbstverständlich vorausgesetzt.

Der Idealfall wäre dort gegeben, wo sich alle drei der oben erwähnten Kriterien kumulativ vorfänden. 
Anmerkungen:

1. Vgl. Frank Barton: African Assignment, The story of IPI's six-year training programme in tropical Africa, Zürich (The International Press Institute) 1969.

2. Vgl. zum Thema: Vernon S. Gerlach: The professional education of the media specialist, in: „Audio Visual Communication Review“, Washington 14:1966, Nr. 2, S. 185 ff.

3. Detlef Bald: Geschichte, Aufgaben und Probleme der Massenmedien im politisch-kulturellen Wandlungsprozeß in Schwarzafrika, in : „Publizistik “, Konstanz, 14:1969, Nr. 1, S. 47-71, hier: S. 53.

4. Detlev Bald, a.a.O. S. 50: Unter den ersten Politikern Nigerias, die für ihr Land die Unabhängigkeit erkämpften, waren mit Macaulay, Ikoli, Awolowo, Azikiwe oder Akintola nur Journalisten und Zeitungsverleger.

5. Vgl. Gottfried Eisermann und S. S. Acquaviva: Massenmedien und sozialer Wandel. Am Beispiel einer unterentwickelten Region, in: „Kölner Zeitschrift für Soziologie und Sozialpsychologie", Opladen, 21:1968, Heft 4.

6. Russel Warren Howe: Journalistenschulung in Afrika, in: „IPI-Rundschau“, Zürich, Nr. 2 von November 1969, S. 17-18, hier: S. 17.

7. Russel Warren Howe, a.a.O. S. 18.

9. Das Ziel dieses Institutes ist die Ausbildung afrikanischer Führungskräfte. Die Kurse dauern zwei Jahre. Weitere Abteilungen existieren für Gemeinschaftsentwicklung und administrative Führungskräfte. Ähnliche Institute gründeten die Weißen Väter in Bukavu, Ost-Kongo, und in Bobo-Dioulasso, Obervolta.

10. Die Beschreibung des Lehrprogramms basiert auf den Plänen für die Kurse der Jahre 1964 und 1965. Die Stundenzahl für die einzelnen Fächer teilt sich in den beiden Unterrichtsjahren wie folgt auf: News reporting (152/104); Editing (90/80); Article writing (41/34); History of the press $(60 /-)$; Science of communications (80/146); Typewrinting (10/-); Photography-theory (10/-). Erstes Jahr: 38 Wochen mit 443 Stunden; zweites Jahr: 26 Wodhen mit 364 Stunden.

11. Die Stundenzahl für die einzelnen Fächer teilt sich in den beiden Unterrichtsjahren wie folgt auf: Social ethics (114/52); Sociology (114/52); Economics (76/52); Community development (38/26); Co-operative movement (38/26); Labour movement (38/26); Political science (-/52); Community service (89/-). Erstes Jahr: 38 Wochen mit 507 Stunden; zweites Jahr: 26 Wochen mit 286 Stunden.

12. Detlev Bald, a.a.O. S. 47.

\section{S U M M A R Y}

In 1963 the Dutch Dipl.-Journalist Piet A. Winnubst founded and managed (from 1963 to 1966) the first school for journalists in Bantu Africa, the "Publicity Media Institute ${ }^{*}$ in Mwanza/Nyegezi (Tanzania). This Training Centre was intended for students from five East and Central African countries. The founders realized, that communication would be the common substratum of development. Moreover they found out that most of the intellectual professions being of some importance for the development of nations were in need of journalistic talents, such as creative thinking, defining, analyzing and communicating. Winnubst made it a rule for his students: Africans should write more. The syllabus of the Nyegezi Centre distinguished between professional training and general education. Today the author does'nt see the least chance for such an engagement from ouside the continent. He enumerates some conditions which generally should be fulfilled by organisations planning schools for journalists in Africa. 